Estela Yadira Reyes-Reyes; José Manuel Piguave-Reyes; Carlos Alberto Riofrío-Pinargote; Mercedes Jahaira Alay-Pinargote

http://dx.doi.org/10.35381/s.v.v4i8.1066

\title{
Evaluación del riesgo biológico en los laboratorios de las Instituciones Universitarias
}

\section{Evaluation of biological risk in the laboratories of University Institutions}

\author{
Estela Yadira Reyes-Reyes \\ estelitareyesreyes@hotmail.com \\ Universidad Laica Eloy Alfaro de Manabí, Manabí \\ Ecuador \\ https://orcid.org/0000-0002-4413-2959 \\ José Manuel Piguave-Reyes \\ Jose.manuel.piguave@hotmail.com \\ Investigador Independiente \\ Ecuador \\ https://orcid.org/0000-0002-6181-0555 \\ Carlos Alberto Riofrío-Pinargote \\ carlosriofrio28@hotmail.com \\ Distrito 13D03 Jipijapa - Puerto López - Salud, Manabí \\ Ecuador \\ https://orcid.org/0000-0001-7837-1563 \\ Mercedes Jahaira Alay-Pinargote \\ jahai 12@hotmail.com \\ Centro de Salud Tipo C Puerto López, Manabí \\ Ecuador \\ https://orcid.org/0000-0002-6499-9007
}

Recepción: 20 de agosto 2020

Revisado: 29 de septiembre 2020

Aprobación: 03 de noviembre 2020

Publicación: 15 de noviembre 2020 
Estela Yadira Reyes-Reyes; José Manuel Piguave-Reyes; Carlos Alberto Riofrío-Pinargote; Mercedes Jahaira Alay-Pinargote

\title{
RESUMEN
}

Las personas que trabajan en laboratorios clínicos están en permanente riesgo de sufrir accidentes laborales por la exposición a material biológico. Con base a esta premisa este artículo se propone como objetivo evaluar el riesgo biológico en los laboratorios clínicos de la universidad estatal del sur de Manabí. Fundamentado en las teorías propuestas por el método BIOGAVAL. La metodologia fue de tipo observacional - descriptiva con un componente exploratorio constituido los usuarios del laboratorio clínico de la Universidad a través de la informacion aportadas a través del instrumento aplicado. Los resultados, permitieron constatar que existe vulnerabilidad de contagio hacia los agentes biológicos que poseen como vía de contaminación la aérea, pues son expansivas y de fácil contacto para alojarse y producir la enfermedad, permitiendo concluir que los mayores factores de riesgos se encontraron el micobacterium tuberculosis, el virus de las hepatitis $C$ y D y el virus de la gripe.

Palabras clave: Transporte biológico; fenómenos biológicos; epidemiología. (Fuente: DeCS, 2020).

\begin{abstract}
People who work in clinical laboratories are at permanent risk of occupational accidents due to exposure to biological material. Based on this premise, this article aims to assess the biological risk in the clinical laboratories of the southern state university of Manabí. Based on the theories proposed by the BIOGAVAL method. The methodology was of an observational-descriptive type with an exploratory component constituted by the users of the University's clinical laboratory through the information provided through the applied instrument. The results made it possible to verify that there is a vulnerability of contagion towards biological agents that have air as a route of contamination, as they are expansive and easy to contact to lodge and produce the disease, allowing to conclude that the greatest risk factors were mycobacterium tuberculosis, the hepatitis $C$ and $D$ virus and the influenza virus.
\end{abstract}

Keywords: Biological transport; biological phenomena; epidemiology. (Source: DeCS, 2020). 
Revista Arbitrada Interdisciplinaria de Ciencias de la Salud. SALUD Y VIDA

Volumen 4. Número 8. Año 4. Julio - Diciembre 2020

Hecho el depósito de Ley: FA2016000010

ISSN: 2610-8038

FUNDACIÓN KOINONIA (F.K)

Santa Ana de Coro, Venezuela.

Estela Yadira Reyes-Reyes; José Manuel Piguave-Reyes; Carlos Alberto Riofrío-Pinargote; Mercedes Jahaira Alay-Pinargote

\section{INTRODUCCIÓN}

El manual BIOGAVAL, aporta un método sencillo y fiable para realizar evaluaciones de riesgos biológicos en actividades donde no se manipulan deliberadamente agentes biológicos, pero en la que los trabajadores se hallan expuestos a los riesgos que se derivan de la presencia de microorganismos, aspecto que limita la aplicación práctica de la guía del Instituto Nacional de Seguridad e Higiene en el Trabajo (INSHT) ${ }^{1}$.

Cabe señalar, que este método se propone facilitar la prevención de los riesgos biológicos, asi como orientar la priorización de las medidas preventivas y de control, todo ello con una inversión de tiempo y dinero relativamente pequeña, especialmente cuando se tiene una cierta práctica en su manejo.

El método BIOGAVAL es un instrumento útil y práctico ${ }^{2}$, que requiere una inversión de tiempo y dinero relativamente baja, especialmente cuando se tiene práctica en su manejo, en las que no se manipulan deliberadamente agentes biológicos, pero existe riesgo de exposición y que contempla el método, tal como el trabajo de los laboratorios clínicos, caso que nos ocupa en el estudio, el mismo consta de los siguientes pasos:

Determinación de puestos a evaluar: puesto de trabajo no equivale forzosamente a categoría profesional, define como las personas realizan tareas homogéneas en un entorno de trabajo común y por tanto presentan una homogeneidad respecto a los riesgos existentes, al grado de exposición y sus graves resultados de un posible daño ${ }^{3}$.

Identificación del agente microbiológico: para este objetivo no es útil la realización de muestreos rutinarios; no hay valores límite establecidos ${ }^{4} \mathrm{y}$ además está desaconsejada por las sociedades científicas de Medicina Preventiva ${ }^{5}$. La identificación se realiza a partir del estudio de los procedimientos de trabajo y de datos epidemiológicos de la población valenciana para identificar aquellas enfermedades que tienen una mayor incidencia (o prevalencia según los casos), puesto que la entrada de los agentes biológicos en el hospital es por medio de los pacientes. No se toman en consideración los agentes microbiológicos del grupo I. Igualmente se excluyen los microorganismos para los cuales 
Estela Yadira Reyes-Reyes; José Manuel Piguave-Reyes; Carlos Alberto Riofrío-Pinargote; Mercedes Jahaira Alay-Pinargote

el trabajo en un hospital no suponga un riesgo adicional de infección con respecto del que tiene el resto de la población, por ejemplo: brucelosis, rabia, tétanos, enfermedades cuya vía de transmisión principal es la sexual.

Cuantificación de la magnitud del riesgo: para ello se considera el daño a la salud que puede causar el microbio (desde unos días de baja laboral, curación con consecuencias permanentes, hasta la muerte del paciente), vías de transmisión (una o varias, privando la vía aérea), ocurrencia de la enfermedad en la población el año anterior, existencia o no de vacuna eficaz y porcentaje de personal expuesto vacunado, frecuencia de las tareas de riesgo y medidas higiénicas aplicadas en esa área o sección según check-list del propio método ${ }^{6}$. El daño producido y la vía de transmisión se verán minimizados por las medidas higiénicas.

Fórmula de cálculo del riesgo: $R=\left(D^{\prime} * V\right)+T^{\prime}+I+F$ siendo: $R=$ nivel de riesgo $D^{\prime}=$ daño - medidas higiénicas $T^{\prime}=$ vía de transmisión - medidas higiénicas $\mathrm{I}=$ tasa de incidencia $\mathrm{F}$ = frecuencia de realización de tareas de riesgo.

El documento incluye también cuatro Anexos: El anexo I, lista orientativa de agentes biológicos que pueden causar enfermedades se presentan asociados a sectores industriales y dentro de éstos, se detallan los microorganismos de riesgo en ramas industriales específicas y la enfermedad que producen. En el anexo II, el listado de vacunas disponibles y en el anexo III, el listado de microorganismos centinela para cada actividad laboral. Los microorganismos centinelas son aquellos presentes habitualmente en la actividad a evaluar y representativos del daño más frecuente capaz de originar. Finalmente en el anexo IV se desarrollan ejemplos prácticos de aplicación del método. 
Revista Arbitrada Interdisciplinaria de Ciencias de la Salud. SALUD Y VIDA

Volumen 4. Número 8. Año 4. Julio - Diciembre 2020

Hecho el depósito de Ley: FA2016000010

ISSN: 2610-8038

FUNDACIÓN KOINONIA (F.K)

Santa Ana de Coro, Venezuela.

Estela Yadira Reyes-Reyes; José Manuel Piguave-Reyes; Carlos Alberto Riofrío-Pinargote;

Mercedes Jahaira Alay-Pinargote

\section{Agentes biológicos}

Se tiene la concepción de que los agentes causales de las enfermedades contagiosas son de la cadena epidemiológica, pero en este trabajo se habla del mismo agente pero en el contexto epidemiológico de un laboratorio clínico, entendiendo que la naturaleza del agente biológico es la misma en cualquier contexto. Pueden ser virus, bacterias, hongos, parásitos y priones. la Organización Mundial de la Salud, en su Manual de Bioseguridad en el Laboratorio, los clasifica en cuatro grupos ${ }^{7}$ :

Grupo de Riesgo 1: Microorganismos que tienen pocas probabilidades de producir enfermedades en humanos y animales. Por tanto, el riesgo individual y poblacional es escaso o inexistente.

Grupo de Riesgo 2: Microorganismos que pueden provocar enfermedades humanas o animales. Tienen pocas probabilidades de entrañar un riesgo grave para el personal de laboratorio, la población, el ganado o el medio ambiente. La exposición en el laboratorio puede provocar una infección grave, y su minimización viene dada al prevenir y utilizar medidas terapéuticas eficaces.

Grupo de Riesgo 3: Microorganismos que suelen provocar enfermedades humanas o animales graves, pero que ordinariamente no se propagan de un individuo a otro. Existen medidas preventivas y terapéuticas eficaces. Por tanto, cuentan con riesgo individual elevado y riesgo poblacional bajo.

Grupo de Riesgo 4: Microorganismos que suelen provocar enfermedades graves en el ser humano o los animales y que se transmiten fácilmente de un individuo a otro, directa o indirectamente. Normalmente no existen medidas preventivas y terapéuticas eficaces. Por tanto, el riesgo individual y poblacional es elevado. 
Estela Yadira Reyes-Reyes; José Manuel Piguave-Reyes; Carlos Alberto Riofrío-Pinargote; Mercedes Jahaira Alay-Pinargote

\section{Vías de entrada de los agentes biológicos}

Las principales vías de acceso a nuestro organismo, ${ }^{8}$ por parte de los agentes biológicos, son las siguientes:

Vías respiratorias: Es la principal vía de acceso, ya que podemos respirar los microorganismos en forma de aerosoles. También es un método de transmisión a tener en cuenta entre el personal del laboratorio a través de la tos o los estornudos. Otro mecanismo mediante esta vía es un mal hábito del laboratorio, como el de oler las placas de cultivo para identificar microorganismos.

Vía digestiva: Si el contagio se produce mediante esta vía de acceso, es debido al uso de malas prácticas de laboratorio. Pipetear con la boca, comer o beber en el laboratorio, o llevarse instrumentos del laboratorio a la boca.

Vía parenteral: Se produce a través de pinchazos o cortes con utensilios que han estado en contacto con material biológico.

Vía dérmica: Se produce a través de la piel. Si entra en contacto con agentes biológicos, éstos pueden adherirse a la piel y colonizarla. También pueden aprovechar cortes, arañazos o heridas previas para adentrarse en nuestro organismo. Si la piel ha sido colonizada y se produce una herida, el agente biológico puede acceder al organismo.

Vía mucosa: El agente biológico accede al organismo a través de sus mucosas.

\section{Utilidad de los grupos de riesgo}

Una de las herramientas más útiles de que se dispone para llevar a cabo una evaluación del riesgo microbiológico, es la asignación de los agentes microbiológicos a uno de los grupos de riesgo. Sin embargo, la mera consulta del grupo de riesgo al que pertenece cierto agente no basta para realizar una evaluación del riesgo. La actividad prevista en el laboratorio (tratamiento con ultrasonidos, producción de aerosoles, centrifugación, entre otras). 
Estela Yadira Reyes-Reyes; José Manuel Piguave-Reyes; Carlos Alberto Riofrío-Pinargote; Mercedes Jahaira Alay-Pinargote

\section{Niveles de bioseguridad del laboratorio}

Según la información obtenida durante la evaluación de los riesgos biológicos en el laboratorio, se podrá asignar un nivel de bioseguridad al trabajo previsto. En función del nivel de bioseguridad asignado, se deberá seleccionar el equipo de protección apropiado para el personal y elaborar procedimientos normalizados de trabajo que incorporen otras intervenciones de seguridad en la realización del trabajo ${ }^{9}$.

Cuadro 1. Relación de los grupos de riesgos con los niveles de bioseguridad, las prácticas y el equipo.

\begin{tabular}{|c|c|c|c|c|}
\hline $\begin{array}{l}\text { GRUPO DE } \\
\text { RIESGO }\end{array}$ & $\begin{array}{l}\text { NIVEL DE } \\
\text { BIOSEGURIDAD }\end{array}$ & $\begin{array}{l}\text { TIPO DE } \\
\text { LABORATORIO }\end{array}$ & $\begin{array}{l}\text { PRAÁCIICAS DE } \\
\text { LABORATORIO }\end{array}$ & $\begin{array}{l}\text { EQUIPO DE } \\
\text { SEGURIDAD }\end{array}$ \\
\hline 1 & $\begin{array}{l}\text { Básico } \\
\text { Nivel } 1\end{array}$ & $\begin{array}{l}\text { Enseñanza básica, } \\
\text { investigación }\end{array}$ & TMA & $\begin{array}{l}\text { Ninguno; trabajo en } \\
\text { mesa de laboratorio } \\
\text { al descubierto }\end{array}$ \\
\hline 2 & $\begin{array}{l}\text { Básico } \\
\text { Nivel } 2\end{array}$ & $\begin{array}{l}\text { Servicios de } \\
\text { atención primaria; } \\
\text { diagnóstico, } \\
\text { investigación }\end{array}$ & $\begin{array}{l}\text { TMA y ropa } \\
\text { protectora; } \\
\text { señal de riesgo } \\
\text { biológico }\end{array}$ & $\begin{array}{l}\text { Trabajo en mesa al } \\
\text { descubierto y CSB } \\
\text { para posibles } \\
\text { aerosoles }\end{array}$ \\
\hline 3 & $\begin{array}{l}\text { Contención } \\
\text { Nivel } 3\end{array}$ & $\begin{array}{l}\text { Diagnóstico } \\
\text { especial, } \\
\text { investigación }\end{array}$ & $\begin{array}{l}\text { Prácticas de nivel } 2 \\
\text { más ropa especial, } \\
\text { acceso controlado } \\
\text { y flujo direccional } \\
\text { del aire }\end{array}$ & $\begin{array}{l}\text { CSB además de otros } \\
\text { medios de contención } \\
\text { primaria para todas } \\
\text { las actividades }\end{array}$ \\
\hline 4 & $\begin{array}{l}\text { Contención } \\
\text { máxima } \\
\text { Nivel } 4\end{array}$ & $\begin{array}{l}\text { Unidades de } \\
\text { patógenos } \\
\text { peligrosos }\end{array}$ & $\begin{array}{l}\text { Prácticas de nivel } 3 \\
\text { más cámara de } \\
\text { entrada con cierre } \\
\text { hermético, salida con } \\
\text { ducha y eliminación } \\
\text { especial de residuos }\end{array}$ & $\begin{array}{l}\text { CSB de clase III o trajes } \\
\text { presurizados junto } \\
\text { con CSB de clase II, } \\
\text { autoclave de doble } \\
\text { puerta (a través de la } \\
\text { pared), aire filtrado }\end{array}$ \\
\hline
\end{tabular}

TMA: técnicas microbiológicas apropiadas.

CSB: cámara de seguridad biológica.

Fuente: Manual de Bioseguridad en el Laboratorio de la OMS. 
Estela Yadira Reyes-Reyes; José Manuel Piguave-Reyes; Carlos Alberto Riofrío-Pinargote; Mercedes Jahaira Alay-Pinargote

En razón de lo presentador se tiene como objetivo del estudio: evaluar el riesgo biológico en los laboratorios clínicos de la universidad estatal del sur de Manabí.

\section{METODOLOGIA}

La metodología fue de tipo observacional descriptivo con un componente exploratorio que incluye la aplicación de un instrumento tipo cuestionario con la finalidad de identificar los principales agentes microbiológicos presente en la institución de estudio.

\section{RESULTADOS}

Para la identificación de los agentes microbiológicos, presentes en los laboratorios clínicos de la Universidad Estatal del sur de Manabí, están basados en los listados de enfermedades de declaración obligatoria y en los agentes biológicos que pueden estar presentes con mayor frecuencia en este medio de trabajo clínico, entre las que se pudo evidenciar con mayores factores de riesgos los siguientes:

a) El Micobacterium tuberculosis, por tener una vía de transmisión aérea ${ }^{10}$.

b) El virus de las hepatitis C y D por su alta gravedad de daño ${ }^{11}$.

c) El virus de la gripe dado el alto grado de presencia en la población usuaria de los laboratorios ${ }^{12}$.

d) Se puede advertir, que entre las causas de la frecuencia de las patologías de la tuberculosis, hepatitis y gripe es la inexistencia de vacunas eficientes para cada caso.

e) Se constató mayor riesgo durante el uso de materiales punzantes y cortantes, al determinarse que en una gran parte de los usuarios había desconocimiento de las prevenciones que se han de tomar durante su uso y manejo, y en otros casos aun conociéndolas no las consideran. 
Estela Yadira Reyes-Reyes; José Manuel Piguave-Reyes; Carlos Alberto Riofrío-Pinargote; Mercedes Jahaira Alay-Pinargote

f) Existe vulnerabilidad de contagio hacia los agentes biológicos que poseen como vía de contaminación la aérea, pues son expansivas y de fácil contacto para alojarse y producir la enfermedad.

g) Es necesario, que los usuarios del laboratorio clínico utilicen como medidas de prevención gafas y guantes. Tambien se debe resaltar de forma visible las recomendaciones a seguir en el laboratorio para disminuir los riesgos biológicos.

h) Se pudo constatar, que aún existen usuarios que no conocen los procedimientos de gestión de los residuos, ni el nivel de peligrosidad de los mismos durante su uso, manejo y desecho.

i) Los usuarios de los laboratorios clínicos, dada la alta movilidad de los mismos están sometidos a un riesgo tal, que no puede considerarse ligado a las condiciones de seguridad de una única área y donde las actividades realizadas no varían sustancialmente de una sección a otra, puede considerarse que tampoco variará la frecuencia de exposición a los distintos agentes biológicos.

j) Por el contrario, la probabilidad determinada por la valoración de las medidas higiénicas, sí que variará ligeramente dependiendo del área donde se encuentre el usuario, para lo cual se tomó la probabilidad más elevada, es decir se consideró la situación más desfavorable. 
Estela Yadira Reyes-Reyes; José Manuel Piguave-Reyes; Carlos Alberto Riofrío-Pinargote;

Mercedes Jahaira Alay-Pinargote

\section{CONCLUSIONES}

El uso del método BIOGAVAL, permitió evaluar los riesgos biológicos en los laboratorios clínicos de la universidad estatal del sur de Manabí. Durante su aplicación, se constató que la mayor frecuencia del riesgos se presentan para los virus del Micobacterium tuberculosis, de la hepatitis C y D y de la gripe ${ }^{13} 1415$.

El inadecuado uso de los materiales punzantes y cortantes, se indicaron con los de mayor riesgo dado, en la mayoría de los casos, por el desconocimiento o alteración de las normas a seguir para su manejo y uso.

Se indican poco uso de los guantes, gafas y otros instrumentales de prevención durante las jornadas de trabajo en el laboratorio clínico, generando un aumento en los riesgos en las tareas propias y prácticas en los laboratorios de la universidad.

\section{CONFLICTO DE INTERÉS}

Los autores declaran que no tienen conflicto de interés en la publicación de este artículo.

\section{FINANCIAMIENTO}

Autofinanciado.

\section{AGRADECIMIENTO}

A la Universidad Laica Eloy Alfaro de Manabí; por apoyar el desarrollo la investigación. 
Estela Yadira Reyes-Reyes; José Manuel Piguave-Reyes; Carlos Alberto Riofrío-Pinargote; Mercedes Jahaira Alay-Pinargote

\section{REFERENCIAS}

1. Fernández-Sánchez, L. Guía técnica para la evaluación y prevención de los riesgos relacionados con la con la exposición a agentes biológicos. REAL DECRETO 664/1997, de 12 de mayo. BOE no 124, de 24 de mayo. 2001 [Internet]. https://n9.cl/ra3ov

2. Contreras-Velásquez Z, Ramírez-Leal P. Comparación de métodos utilizados en la valoracion del riesgo biológico [Comparative study of methods for the valoration of biological risk]. Rev Asoc Esp Espec Med Trab [Internet]. 2019; 28( 2 ): 91-108.

3. Benavent-Nácher S, Machí-Alapont M, Moliner-Sales I, Soto-Ferrando P. Evaluación de riesgo biológico en el Hospital Rey Don Jaime. Med. segur. trab. [Internet]. 2007 Mar; 53( 206 ): 9-14.

4. Luna, P. Contaminantes químicos: evaluación de la exposición laboral INSHT, Ministerio de Trabajo y Asuntos Sociales. [Chemical pollutants: evaluation of INSHT labour exposure, Ministry of Labour and Social Affairs]. España 2008

5. Borras Moliner MG. Recomendaciones para el control de la infección nosocomial. Generalitat Valenciana. [Recommendations for the control of nosocomial infection . Generalitat Valenciana Conselleria de Sanitat.2003

6. Dawson B y Trapp, R. Bioestadística médica. [Medical biostatistics]. México: Manual Moderno S.A. Cuarta Edición. p. 392.2005

7. Cortés, J. Técnicas de Prevención de Riesgos Laborales, Seguridad e higiene del trabajo [Occupational Risk Prevention, Occupational Safety and Hygiene Techniques]. España: Editorial Tebar.2011

8. Argimon J y Jiménez J. Métodos de investigación clínica y epidemiológica. [Clinical and epidemiological research methods]. España: Elsevier S.L, 3ra edición.2009

9. Aguilar. E; Campo, R; Morchón, V y Martínez, M. Diferencias de protección frente al riesgo biológico laboral en función del tamaño de la empresa. [Differences in protection against occupational biological risk depending on the size of the company]. Revista de Salud Pública, vol.17, no.2, p.1-1. 2015 
Revista Arbitrada Interdisciplinaria de Ciencias de la Salud. SALUD Y VIDA

Volumen 4. Número 8. Año 4. Julio - Diciembre 2020

Hecho el depósito de Ley: FA2016000010

ISSN: 2610-8038

FUNDACIÓN KOINONIA (F.K).

Santa Ana de Coro, Venezuela.

Estela Yadira Reyes-Reyes; José Manuel Piguave-Reyes; Carlos Alberto Riofrío-Pinargote;

Mercedes Jahaira Alay-Pinargote

10. Koenig SP, Furin J. Update in Tuberculosis/Pulmonary Infections 2015. Am J Respir Crit Care Med. 2016 Jul 15;194(2):142-6. doi: 10.1164/rccm.201601-0129UP. PMID: 27420359; PMCID: PMC5003219.

11. Applegate TL, Fajardo E, Sacks JA. Hepatitis C Virus Diagnosis and the Holy Grail. Infect Dis Clin North Am. 2018 Jun;32(2):425-445. doi: 10.1016/j.idc.2018.02.010. PMID: 29778264.

12. Wiwanitkit V. Influenza infection in ICU [La infección por gripe en la UCI]. Enferm Infecc Microbiol Clin. 2016 Aug-Sep;34(7):468. Spanish. doi: 10.1016/..eimc.2015.06.003. Epub 2015 Jul 8. PMID: 26164265.

13. Pol S, Lagaye S. The remarkable history of the hepatitis $C$ virus. Genes Immun. 2019 May;20(5):436-446. doi: 10.1038/s41435-019-0066-z. Epub 2019 Apr 25. PMID: 31019253.

14.Zeisel MB, Felmlee DJ, Baumert TF. Hepatitis C virus entry. Curr Top Microbiol Immunol. 2013;369:87-112. doi: 10.1007/978-3-642-27340-7_4. PMID: 23463198. Zeisel MB, Felmlee DJ, Baumert TF. Hepatitis C virus entry. Curr Top Microbiol Immunol. 2013;369:87-112. doi: 10.1007/978-3-642-27340-7 4. PMID: 23463198.

15. Chan ST, Ou JJ. Hepatitis C Virus-Induced Autophagy and Host Innate Immune Response. Viruses. 2017 Aug 12;9(8):224. doi: 10.3390/v9080224. PMID: 28805674; PMCID: PMC5580481.

2020 por los autores. Este artículo es de acceso abierto y distribuido según los términos y condiciones de la licencia Creative Commons Atribución-NoComercial-Compartirlgual 4.0 Internacional (CC BY-NC-SA 4.0)

(https://creativecommons.org/licenses/by-nc-sa/4.0/). 